\title{
HUMAN FACTORS LEARNING CURVE FOR THUMB- OPERATED TRIGGER RIFLE USING GYROSCOPIC FEEDBACK INSTRUMENTATION
}

\author{
David C. Paulus, PhD, PE, CHFEP \\ Washington State University, Pullman, WA
}

Corresponding Author: David C. Paulus

Email: davidpaulus@hotmail.com

https://doi.org/10.34107/BiomedSciInstrum.57.04274

\begin{abstract}
Researchers interested in evaluating the biomechanics and human factors associated with using a new product recognize that skill development with the novel design is time-dependent. A learning curve is a plot that shows the time to complete a task using the product decreases as the number of training repetitions increases. A novel thumb-operated trigger system (Iron Horse, Blackwater Worldwide ${ }^{\mathrm{TM}}$ ) has been developed for the AR-15 style rifle with the intent to shorten the learning curve. The purpose of this research effort is to quantify the learning curve for the new device and to compare it to that of a standard mil-spec AR-15 trigger system. A previously-trained shooter dry-fire trained with both rifle systems for twenty consecutive days alternating lower receivers each day. The rifles were equipped with a gyroscopic instrument (Mantis $\mathrm{X}^{\mathrm{TM}}$ ) that tracked the movement of the firearm during the trigger pull process. The instrument has a timer to record the reaction time to an auditory signal for each shot, records the magnitude and direction of movement of the firearm, and calculates an accuracy score. There was not a significant difference $(\mathrm{p}>0.05)$ between the thumb operated and mil-spec triggers' cycle times. However, the accuracy scores with the thumb operated trigger were significantly higher $(\mathrm{p}<0.05)$ than those with the mil-spec trigger.
\end{abstract}

Keywords: Dry-Fire, MantisX, Training, Reaction Time

\section{INTRODUCTION}

Human factors is the study of how humans interact with their environment. Training has been extensively studied in the field of human factors and is a standard procedure used to help people acquire new skills [1]. Successful training depends on (a) instruction: positive approaches that stress the learning of new behaviors (b) practice: suitable conditions for practice that ensure the transferability of these learned behaviors to the real settings, and (c) feedback: the inclusion of means for evaluating their effectiveness in reaching specified performance goals with frequent feedback [1,2]. Firearms training has typically required a qualified instructor to provide feedback of shooting technique. However, Mantis Tech LLC (Oswego, IL) developed a technology called the MantisX that tracks the movement of the firearm during the trigger pull and provides feedback after each shot. The technology uses gyroscopic angle measurement and works with a smartphone/tablet application via Bluetooth. The application provides the direction and magnitude of movement, a shot-by-shot analysis, and a detailed trace with a blue line for hold/ sighting, a yellow line for the trigger pull, and a red line showing shot breaking and recoil pattern. Figure 1 shows examples of the MantisX feedback. 